\title{
Asociación entre soporte familiar y control glicémico en pacientes con diabetes mellitus de tipo 2 en un Consultorio de Endocrinología de un hospital nacional
}

\author{
Carlos Abuhadba Miranda * 1,a ; Nora Espíritu 1,2,b; Dante Gamarra Gonzáles 2,3,c
}

\section{RESUMEN}

Objetivo: Determinar la asociación entre soporte familiar y control glicémico en pacientes con diabetes mellitus tipo 2 que acuden al Consultorio Externo de Endocrinología del Hospital Nacional Dos de Mayo.

Materiales y métodos: Se realizó un estudio cuantitativo, observacional y transversal. La muestra fue de 158 sujetos diabéticos mayores de 18 años con hemoglobina glicosilada al momento de la consulta, obtenida mediante Epi info v7.9.2, con un nivel de confianza $95 \%$, precisión del $5 \%$ y con una frecuencia esperada de $12,11 \%$. El muestreo fue no probabilístico por conveniencia. Se aplicó una ficha de recolección, registrándose datos sociodemográficos, clínicos y del entorno social del paciente, así como una encuesta de la Escala de Cohesión y Adaptabilidad Familiar-FACES III para determinar el soporte familiar. Se realizó la prueba chi cuadrado para determinar la asociación entre soporte familiar y control glicémico.

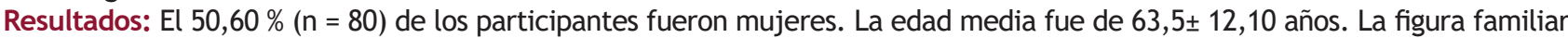
más representativa de cuidador primario fue el hijo en un $50,6 \%(n=80)$ seguido del cónyuge $20,3 \%(n=32)$. De los 158 sujetos, $98(62 \%)$ tuvieron control glicémico controlado y de estos $44(44,9 \%)$ un soporte familiar adecuado. De los 60 no controlados, $16(26,6 \%)$ tuvieron un soporte familiar adecuado. Se determinó la asociación entre soporte familiar y control glicémico siendo estadísticamente significativa $\mathrm{X}^{2}=5,252$, con un $\rho<0,05$.

Conclusiones: Existe una asociación significativa entre el soporte familiar y el control glicémico en los pacientes diabéticos que se atienden en los consultorios de endocrinología del Hospital Nacional Dos de Mayo.

Palabras clave: Diabetes mellitus; Familia; Hemoglobina A glucada; Apoyo social; Glucemia (Fuente: DeSC BIREME).

\section{Association between family support and glycemic control among patients with type 2 diabetes mellitus who attend an endocrinology office of a public hospital}

\author{
ABSTRACT
}

Objective: To determine the association between family support and glycemic control among patients with type 2 diabetes mellitus who attend the outpatient endocrinology office of the Hospital Nacional Dos de Mayo.

Materials and methods: A quantitative, observational and cross-sectional study was carried out. The sample consisted of 158 diabetic subjects over 18 years of age whose glycosylated hemoglobin was obtained at the time of the consultation using the Epi Info version 7.9.2 software, with a $95 \%$ confidence level, $5 \%$ accuracy and $12.11 \%$ expected frequency. A non-probability convenience sampling was used. A data collection sheet was employed to register the patients' sociodemographic, clinical and social environment data, as well as the Family Adaptability and Cohesion Evaluation Scale III (FACES III) to determine the family support. A chi-square test was used to determine the association between family support and glycemic control.

Results: Female patients accounted for $50.6 \%(n=80)$ of the sample. The mean age was $63.5 \pm 12.10$ years. The patients' children $(50.6 \%, n=80)$ and spouses $(20.3 \%, n=32)$ were the most likely primary caregivers. Out of the 158 study subjects, 98 (62\%) had a good glycemic control, $44 \%$ of whom (44.9\%) presented an adequate family support. Out of the 60 patients with no glycemic control, $16(26.6 \%)$ had an adequate family support. A statistically significant association between family support and glycemic control was demonstrated, with $\mathrm{X}^{2}=5,252$ and $\rho$ value $<0.05$.

Conclusions: There is a significant association between family support and glycemic control among the diabetic patients treated at the outpatient endocrinology office of the Hospital Nacional Dos de Mayo.

Keywords: Diabetes mellitus; Family; Glycated hemoglobin A; Social support; Blood glucose (Source: MeSH NLM).

1 Universidad San Martin de Porres, Facultad de Medicina Humana. Lima, Perú.

2 Hospital Nacional Dos de Mayo. Lima, Perú.

3 Universidad Nacional Mayor de San Marcos, Facultad de Medicina Humana. Lima, Perú.

a Estudiante de Medicina.

b Médica pediatra, Magister en Salud Pública.

c Médico endocrinólogo, Magister en Docencia e Investigación en Salud.

* Autor corresponsal. 


\section{INTRODUCCIÓN}

La diabetes mellitus tipo 2 es una enfermedad cuya prevalencia e incidencia ha ido aumentando en los últimos años, es causa de múltiples comorbilidades y ocasiona altas tasas de mortalidad y discapacidad. Las principales comorbilidades de la diabetes son la ceguera, insuficiencia renal crónica, infarto de miocardio, accidente cerebro vascular y amputación de miembros inferiores.

En el 2015, la diabetes fue la causa directa de 1,6 millones de muertes; de las cuales, la mitad corresponden a pacientes menores de 70 años. Se estima que para el 2045, los casos de diabetes aumentarían a 693 millones más, y para el 2030, la Organización Mundial de la Salud (OMS) pronostica que será la séptima causa de mortalidad en todo el mundo ${ }^{(1)}$. En el Perú, se estima que 6,7 \% de personas mayores de 18 años tienen hiperglicemia (niveles mayores o iguales a $126 \mathrm{mg} / \mathrm{dl}$ ) o que toman hipoglucemiantes 0 tuvieron un diagnóstico previo de diabetes mellitus ${ }^{(2)}$. Un reporte de PERUDIAB, en el cual se entrevistó a personas mayores de 25 años en áreas urbanas y suburbanas, encontró una prevalencia de diabetes mellitus del $7 \%$. De este porcentaje, el $60 \%$ de los encuestados refirió que un médico o una enfermera les informó que padecían diabetes o utilizaban medicación para tratarla (3). La Dirección General de Epidemiología señala que esta enfermedad es la sexta causa de carga de enfermedad en el país, y la primera en personas de 45 a 59 años ${ }^{(4,5)}$.

En el Hospital Nacional Arzobispo Loayza, GonzalesGrández et al. encontraron que el $70 \%$ de los ingresos en pacientes diabéticos fueron por infecciones, como urinarias, pie diabético, respiratorias, de partes blandas, etc., y el $24 \%$, por complicaciones metabólicas agudas como hipoglucemias, cetoacidosis diabética, estado hiperosmolar, etc. ${ }^{\left({ }^{6}\right)}$. Por todo esto, la diabetes resulta ser una enfermedad costosa, tanto a nivel económico como en la calidad de vida. Una buena educación del paciente y de su entorno social es necesaria para conseguir buena adherencia al tratamiento, controlar la enfermedad y limitar sus comorbilidades.

En diferentes estudios se ha descrito que el entorno familiar influye directamente en el desarrollo del individuo en sus distintas facetas. En el cuidado de la salud, se ha demostrado que la familia cumple un rol de gran trascendencia, especialmente si hablamos de la protección y la conservación de la salud. Es así como la salud de la familia está influenciada por vivencias, conflictos, acontecimientos de la vida y relaciones interpersonales, actuando todas estas directamente sobre la salud de los integrantes del grupo familiar ${ }^{(7)}$.

Dada la importancia del ambiente familiar y las herramientas que este proporciona al paciente para el afrontamiento de problemas y situaciones adversas, se plantea esta investigación con el objetivo de determinar la asociación entre control glicémico y niveles de soporte social en pacientes con diabetes mellitus tipo 2 que acuden al Consultorio Externo de Endocrinología del Hospital Nacional Dos de Mayo.

\section{MATERIALES Y MÉTODOS}

\section{Diseño y población de estudio}

Estudio cuantitativo, observacional y transversal. En la investigación se entrevistó a pacientes diagnosticados con diabetes mellitus tipo 2 que se atienden en el Consultorio Externo de Endocrinología del Hospital Nacional Dos de Mayo, en donde se atendieron 3153 pacientes en 2018. Se obtuvo una muestra de 158 pacientes. El muestreo fue no probabilístico por conveniencia. Se empleó el software estadístico Epi info versión 7.9.2, con un nivel de confianza del $95 \%$, una precisión del $5 \%$ y una frecuencia esperada del $12,11 \%{ }^{(8)}$.

\section{Variables y mediciones}

Se consideraron variables sociodemográficas como edad, sexo, procedencia, nivel de instrucción y ocupación; y variables clínicas como tiempo de enfermedad de la diabetes, presencia de comorbilidades y complicaciones. La cohesión y adaptabilidad familiar fueron medidas con la Encuesta de Cohesión y Adaptabilidad Familiar FACIES III, cuyo resultado nos indica el tipo de soporte familiar como adecuado o no adecuado. Así también se consideraron como variables la asistencia al programa de diabetes y el tipo de cuidador primario. La variable control glicémico que maneja el Consultorio de Endocrinología del HNDM, se basa en los objetivos de control glicémico individualizado según la Guía de diabetes tipo 2 para clínicos: recomendaciones de la red GDPS $2018{ }^{(9)}$, cuyos criterios están planteados en función de la edad del sujeto, duración de la diabetes, presencia de complicaciones o comorbilidades y de la hemoglobina glicosilada objetivo. 
Asociación entre soporte familiar y control glicémico en

pacientes con diabetes mellitus de tipo 2 del Consultorio de

Endocrinología de un hospital nacional

Tabla 1. Objetivo de control glicémico individualizado de la Guía de diabetes tipo 2 para clínicos: recomendaciones de la red GDPS 2018

\begin{tabular}{clr} 
Edad & Duración de la DM, presencia de complicaciones o comorbilidades & HbA1c objetivo \\
\hline$\leq 65$ años & Sin complicaciones o comorbilidades graves & $<7,0 \%{ }^{*}$ \\
& $>15$ años de evolución o con complicaciones o comorbilidades graves & $<8,0 \%$ \\
\hline $66-75$ años & $\leq 15$ años de evolución sin complicaciones o comorbilidades graves & $<7,0 \%$ \\
& $>15$ años de evolución sin complicaciones o comorbilidades graves & $<, 0-8,0 \%$ \\
\hline & Con complicaciones o comorbilidades graves & $<, 5 \% \%^{* *}$
\end{tabular}

* Puede plantearse un objetivo de $\mathrm{HbA} 1 \mathrm{c} \leq 6,5 \%$ en los pacientes más jóvenes y de corta evolución de la diabetes en tratamiento no farmacológico o con monoterapia.

** No se debe renunciar al control de los síntomas de hiperglucemia, independientemente del objetivo de HbA1c.

Se incluyeron a todos los pacientes mayores de 18 años, orientados en tiempo, espacio y persona, con diagnóstico de diabetes mellitus tipo 2, que tenían hemoglobina glicosilada al momento de la consulta. Se excluyeron a los pacientes que no respondieron completamente la encuesta, que no llenaban correctamente sus datos y que tuvieran trastornos cognitivos o psicológicos además de discapacidades físicas que interfirieran con su participación.

El instrumento que se aplicó consta de dos partes: la primera es una ficha de recolección en la que se registraron datos sociodemográficos, datos de la enfermedad y entorno social del paciente, definiendo como cuidador primario a aquella persona que aporta el máximo apoyo instrumental, afectivo y emocional al paciente ${ }^{\left({ }^{9}\right)}$. La segunda parte es la encuesta de la Escala de Cohesión y Adaptabilidad Familiar-FACIES III, diseñada por David Olson, Joyce Portner y Yoav Lavee de la Universidad de Minnesota en 1985, validada para la población peruana. Tiene 20 ítems en una escala de Likert del 1 al 5 , donde (1) es Casi nunca o Nunca, (2) es De vez en cuando, (3) es A veces, (4) es Muchas veces y (5) es Casi siempre o Siempre.

La confiabilidad de la encuesta de la Escala de Cohesión y Adaptabilidad Familiar FACIES III (10) para la escala de cohesión es $r=0,77$ y para adaptabilidad es $r=0,62$, lo cual da un total de $r=0,68$. En cuanto a la validez, la correlación entre las escalas de cohesión y adaptabilidad es de $r=0,03$, la correlación con deseabilidad social (DS) es para adaptabilidad $r=000$ como para cohesión $r=0,39$, y en la validez concurrente no se halló evidencia ${ }^{(11)}$.

\section{Análisis estadístico}

Los datos obtenidos en las encuestas se ingresaron al programa de hoja de cálculo Microsoft Excel versión 2016. Luego de completar la base de datos se procedió a un control de calidad para identificar y corregir errores, y encontrar vacíos o valores anormales en las variables. Para ello, dos digitadores revisaron más de una vez la base de datos.
Para el análisis se utilizó el programa IBM SPSS Statistics 25. Al ser un estudio que compara los resultados de dos variables, el análisis fue bivariado, y se empleó la prueba de ji al cuadrado $\left(x^{2}\right)$.

\section{Consideraciones éticas}

El protocolo fue aprobado por el Comité de Ética en Investigación de la Facultad de Medicina Humana de la Universidad de San Martin de Porres y por el CIEl del Hospital Nacional Dos de Mayo. En este estudio se respetaron las normas de la buena práctica clínica y se cumplieron las normas éticas estipuladas en la declaración de Helsinki. Se solicitó el consentimiento informado a los sujetos de investigación previa su participación, sus nombres fueron codificados para mantener el anonimato.

\section{RESULTADOS}

Se encuestaron a 158 sujetos durante el mes de enero y febrero del 2020. El 50,60 \% $(n=80)$ fueron mujeres y el $49,40 \%(n=78)$, hombres. La edad media fue de 63,5 años con una desviación estándar de 12,10 años.

El 54,00\% ( $n=86)$ fueron menores de 65 años, 32,00\% $(n=50)$ tenían entre $66-75$ años, y $14,00 \%(n=22)$, más de 75 años. La ocupación que predominó fue la de ama de casa con un $41,80 \%(n=66)$, seguida de trabajador independiente $(30,40 \%)$ y jubilado $(12,70 \%)$. El nivel de instrucción más común fue la educación secundaria con un $46,80 \%(n=74)$, luego la educación primaria $(33,50 \%)$, superior técnico $(10,10 \%)$, pregrado universitario $(8,90 \%)$ y posgrado universitario (solo un encuestado). El distrito del cual provenía el mayor número de encuestados fue La Victoria (25,30\%; $n=40)$, le siguieron el Cercado de Lima $(23,40 \%)$ y San Juan de Lurigancho $(17,10 \%)$. Todos ellos son distritos colindantes al hospital. Como cuidador primario, el familiar más representativo fue el hijo para 80 de los participantes (50,60\%), y luego el cónyuge, con el 20,30\% ( $\mathrm{n}=32)$ (Tabla 2). 
Tabla 2. Características sociodemográficas de los pacientes encuestados en el Consultorio de Endocrinología en el Hospital Nacional Dos de Mayo. Lima, Perú

\begin{tabular}{|c|c|c|}
\hline $\begin{array}{l}\text { Características } \\
\text { sociodemográficas }\end{array}$ & $\begin{array}{c}N=158 \\
n\end{array}$ & $\begin{array}{c}\text { Porcentaje } \\
(\%)\end{array}$ \\
\hline Sexo & & Sexo \\
\hline Mujer & 80 & $(50,60)$ \\
\hline Hombre & 78 & $(49,40)$ \\
\hline \multicolumn{3}{|l|}{ Rango de edad } \\
\hline$\leq 65$ años & 86 & $(54,00)$ \\
\hline 66-75 años & 50 & $(32,00)$ \\
\hline > 75 años & 22 & $(14,00)$ \\
\hline \multicolumn{3}{|l|}{ Ocupación } \\
\hline Ama de casa & 66 & $(41,80)$ \\
\hline Trabajador independiente & 48 & $(30,40)$ \\
\hline Jubilado & 20 & $(12,70)$ \\
\hline Asalariado & 14 & $(8,90)$ \\
\hline Desempleado & 9 & $(5,70)$ \\
\hline Estudiante & 1 & $(0,50)$ \\
\hline \multicolumn{3}{|l|}{ Nivel de instrucción } \\
\hline Primaria & 53 & $(33,50)$ \\
\hline Secundaria & 74 & $(46,80)$ \\
\hline Superior técnico & 16 & $(10,10)$ \\
\hline Pregrado universitario & 14 & $(8,90)$ \\
\hline Postgrado universitario & 1 & $(0,70)$ \\
\hline \multicolumn{3}{|l|}{ Distrito de procedencia } \\
\hline La Victoria & 40 & $(25,30)$ \\
\hline Cercado de Lima & 37 & $(23,40)$ \\
\hline San Juan de Lurigancho & 27 & $(17,10)$ \\
\hline Ate & 7 & $(4,40)$ \\
\hline El Agustino & 6 & $(3,80)$ \\
\hline San Martín de Porres & 4 & $(2,50)$ \\
\hline Otros & 37 & $(23.50)$ \\
\hline \multicolumn{3}{|l|}{ Cuidador primario } \\
\hline Hijos & 80 & $(50,60)$ \\
\hline Cónyuge & 32 & $(20,20)$ \\
\hline Conviviente & 9 & $(5,70)$ \\
\hline Progenitor & 6 & $(3,80)$ \\
\hline Nieto & 1 & $(0,70)$ \\
\hline Otros & 30 & $(19,00)$ \\
\hline
\end{tabular}

El $70,30 \%$ de los pacientes tenían un tiempo de enfermedad menor o igual a 15 años $(n=111)$, y el $29,70 \%$ era mayor de 15 años. El promedio de tiempo de enfermedad fue de 11 años, con una desviación estándar de 9,51 .
Los pacientes con múltiples complicaciones fueron las más numerosos con 20,3 \% (32 casos), seguido de las alteraciones neurológicas $(18,40 \% ; n=29)$, las complicaciones oftalmológicas $(8,20 \%)$, las circulatorias periféricas $(4,40 \%$, y las renales $(3,8 \%)$. El 43,60\% $(n=69)$ de los pacientes no tuvieron complicaciones (Tabla 3).

Tabla 3. Tipo de complicaciones más frecuentes en los pacientes encuestados en el Consultorio de Endocrinología en el Hospital Nacional Dos de Mayo. Lima, Perú

\begin{tabular}{|c|c|c|}
\hline $\begin{array}{c}\text { Tipo de } \\
\text { complicaciones }\end{array}$ & $\begin{array}{c}N=158 \\
n\end{array}$ & $\begin{array}{c}\text { Porcentaje } \\
(\%)\end{array}$ \\
\hline Sin mención & 69 & $(43,60)$ \\
\hline Múltiples & 32 & $(20,30)$ \\
\hline Neurológicas & 29 & $(18,40)$ \\
\hline Oftalmológicas & 13 & $(8,20)$ \\
\hline Circulatorias periféricas & 7 & $(4,40)$ \\
\hline Renales & 6 & $(3,80)$ \\
\hline No especificadas & 2 & $(1,30)$ \\
\hline Total & 158 & $(100)$ \\
\hline
\end{tabular}

La tabla 4 muestra las comorbilidades de los pacientes: la hipertensión arterial fue la más frecuente $(21,50 \% ; n=34)$, luego el hipotiroidismo (5,10\%), las dislipidemias $(3,80 \%)$ $y$ otras $(12,00 \%)$. Mientras que el 20,90\% $(n=33)$ de los encuestados tuvo múltiples comorbilidades.

Tabla 4. Tipo de comorbilidades en los pacientes encuestados en el Consultorio de Endocrinología en el Hospital Nacional Dos de Mayo. Lima, Perú

\begin{tabular}{|lrr|}
\hline \multicolumn{1}{c}{ Tipo de } & $\begin{array}{c}\text { N=158 } \\
n\end{array}$ & $\begin{array}{c}\text { Porcentaje } \\
(\%)\end{array}$ \\
\hline Sin comorbilidades & 58 & $(36,70)$ \\
\hline Hipertensión arterial & 34 & $(21,50)$ \\
\hline Múltiples comorbilidades & 33 & $(20,90)$ \\
\hline Hipotiroidismo & 8 & $(5,10)$ \\
\hline Dislipidemias & 6 & $(3,80)$ \\
\hline Otras & 19 & $(12,00)$ \\
\hline
\end{tabular}


Asociación entre soporte familiar y control glicémico en pacientes con diabetes mellitus de tipo 2 del Consultorio de Endocrinología de un hospital nacional

De los 158 pacientes encuestados, 98 (62,00\%) tuvieron un control glicémico controlado y de estos $44(44,9 \%)$ un soporte familiar adecuado. De los 60 no controlados, $16(26,6 \%)$ tuvieron un soporte familiar adecuado. Se determinó la asociación entre soporte familiar y control glicémico mediante la prueba de ji al cuadrado, que fue estadísticamente significativa $\mathrm{X}^{2}=5,252$, con un $\rho=0,022$ (Tabla 5).

Tabla 5. Tipo de comorbilidades en los pacientes encuestados en el Consultorio de Endocrinología en el Hospital Nacional Dos de Mayo. Lima, Perú

\begin{tabular}{|c|c|c|c|c|c|c|c|c|}
\hline \multirow{3}{*}{ Soporte familiar } & \multicolumn{4}{|c|}{ Control Glicémico } & \multirow{2}{*}{\multicolumn{2}{|c|}{ Total }} & \multirow{3}{*}{$\mathrm{X}^{2}$} & \multirow{3}{*}{$\rho$} \\
\hline & \multicolumn{2}{|c|}{ Controlado } & \multicolumn{2}{|c|}{ No controlado } & & & & \\
\hline & $\mathrm{n}$ & $(\%)$ & $n$ & $(\%)$ & $\mathrm{n}$ & (\%) & & \\
\hline Adecuado & 44 & $(27,8)$ & 16 & $(10,2)$ & 60 & (38) & 5,252 & 0,022 \\
\hline No adecuado & 54 & $(34,2)$ & 44 & $(27,8)$ & 98 & $(62)$ & & \\
\hline Total & 98 & $(62,0)$ & 60 & $(38,0)$ & 158 & (100) & & \\
\hline
\end{tabular}

$X^{2}=5,252 / \rho=0,022$

Según el rango de edad, el grupo que mostró un mejor control glicémico fue el de los menores de 66 años (44,90\%), seguido del grupo de 66 a 75 años (38,80\%) y por el de los mayores de $75(16,30 \%)$. La variable "rango de edad" tuvo una asociación significativa con el control glicémico siendo la prueba $X^{2}=9,524$, con un $\rho=0,009$ (Tabla 6).

Tabla 6. Asociación entre control glicémico y rango de edad en el Consultorio de Endocrinología del Hospital Nacional Dos de Mayo

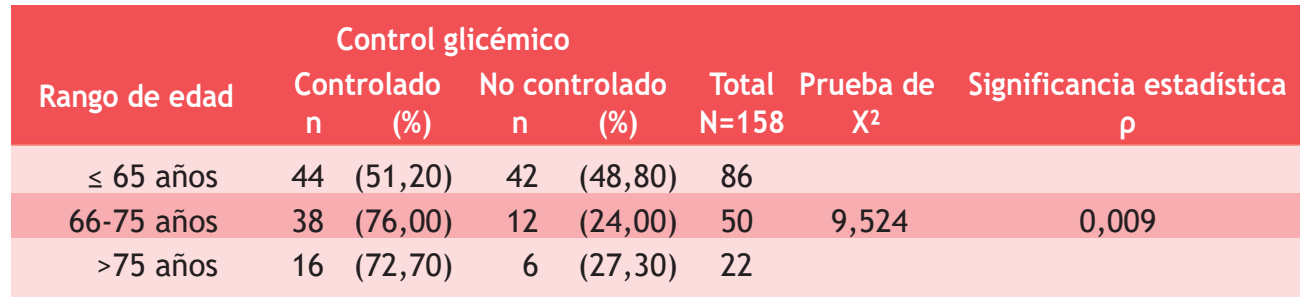

Además de ello, 40 (25,30\%) de los 158 sujetos evaluados asisten regularmente al área de diabetes, de estos, 27 $(67,50 \%)$ tienen un control adecuado de la glicemia. De los 98 con control glicémico adecuado, 69 (70,40\%) tienen un tiempo de enfermedad menor o igual a 15 años. De los 98 pacientes adecuadamente controlados, 54 (55,10\%) tienen complicaciones. Mientras que del grupo de 96 encuestados con comorbilidades, el 61,22 \% $(n=60)$ controlan bien la glicemia (Tabla 7).

Tabla 7. Control glicémico según diversos factores en los pacientes encuestados en el Consultorio de Endocrinología en el Hospital Dos de Mayo

\begin{tabular}{|c|c|c|c|c|c|}
\hline \multirow[b]{2}{*}{ Factores diversos } & \multicolumn{3}{|c|}{ Control glicémico } & \multirow[b]{2}{*}{$\begin{array}{c}\text { Total } \\
\mathrm{N}=158\end{array}$} & \multirow[b]{2}{*}{$\begin{array}{c}\text { Porcentajes } \\
(\%)\end{array}$} \\
\hline & $\begin{array}{l}\text { Controlado } \\
\mathrm{n}=98 \quad(\%)\end{array}$ & $\begin{array}{c}\text { No con } \\
n=60\end{array}$ & $\begin{array}{l}\text { trolado } \\
\qquad(\%)\end{array}$ & & \\
\hline \multicolumn{6}{|c|}{ Asiste regularmente al Área de Diabetes } \\
\hline Sí & $27 \quad(27,50)$ & 13 & $(21,70)$ & 40 & $(25,30)$ \\
\hline No & $71 \quad(72,50)$ & 47 & $(78,30)$ & 118 & $(74,70)$ \\
\hline \multicolumn{6}{|c|}{ Tiempo de enfermedad por rangos } \\
\hline$\leq 15$ años & $69 \quad(70,40)$ & 42 & $(70,00)$ & 111 & $(70,30)$ \\
\hline >15 años & $29 \quad(29,60)$ & 18 & $(30,00)$ & 47 & $(29,70)$ \\
\hline
\end{tabular}




\begin{tabular}{|c|c|c|c|c|c|}
\hline \multirow[b]{2}{*}{ Factores diversos } & \multicolumn{3}{|c|}{ Control glicémico } & \multirow[b]{2}{*}{$\begin{array}{c}\text { Total } \\
\mathrm{N}=158\end{array}$} & \multirow[b]{2}{*}{$\begin{array}{c}\text { Porcentajes } \\
\text { (\%) }\end{array}$} \\
\hline & $\begin{array}{l}\text { Controlado } \\
\mathrm{n}=98 \quad(\%)\end{array}$ & $\begin{array}{c}\text { No con } \\
n=60\end{array}$ & $\begin{array}{c}\text { trolado } \\
\text { (\%) }\end{array}$ & & \\
\hline \multicolumn{6}{|c|}{ Presencia de complicaciones } \\
\hline Sí & $54 \quad(55,10)$ & 35 & $(58,30)$ & 89 & $(56,30)$ \\
\hline No & $44 \quad(44,90)$ & 25 & $(41,70)$ & 69 & $(43,70)$ \\
\hline \multicolumn{6}{|c|}{ Presencia de comorbilidades } \\
\hline Sí & $60 \quad(61,22)$ & 36 & $(60,00)$ & 96 & $(60,80)$ \\
\hline No & $38 \quad(38,78)$ & 24 & $(40,00)$ & 62 & $(39,20)$ \\
\hline
\end{tabular}

No se encontró asociación estadísticamente significativa entre instrucción, cuidador primario y control glicémico.

\section{DISCUSIÓN}

En la última década, la diabetes ha experimentado un aumento notable en su prevalencia. La edad media de los encuestados es de 63,5 años, semejante a la encontrada en el estudio mexicano de García-Morales, en el cual señalan que tuvieron una media de 61,5 años. Cuando realizamos la evaluación de la asociación entre el soporte familiar y el control glicémico, encontramos una asociación estadísticamente significativa, lo cual difiere con resultados de García-Morales, Sánchez Basilio y otros autores ${ }^{(12-15)}$. Esta diferencia podría deberse a que en el estudio García-Morales el $60 \%$ de los pacientes encuestados tenían un mal control glicémico, a diferencia de nuestro estudio, que reporta solo $40 \%$ de mal control glicémico; y respecto a la ocupación, la más prevalente es la de ama de casa para ambos reportes ${ }^{(12)}$. Por otro lado, en el estudio de Sánchez Basilio se realizó el control glicémico basándose en un único corte de hemoglobina glicosilada (>7\%), en contraste con este estudio, que adaptó el punto corte para la hemoglobina glicosilada de acuerdo a la edad del paciente, los años de enfermedad y presencia de comorbilidades y/o complicaciones ${ }^{(13)}$.

Casi la mitad de los sujetos son del sexo masculino, semejante a lo descrito por Seclen en su reporte del año 2015 sobre PERUDIAB ${ }^{(3)}$, pero difiere con los hallazgos de Espínola Chamorro en su tesis de grado, que encontró que el $71,13 \%$ de los participantes eran varones (población paraguaya). También existe una similitud en cuanto a la alta prevalencia de la enfermedad en personas con bajo nivel de instrucción. El rango de edad en donde hay una mayor cantidad de pacientes diagnosticados es en menores de 65 años, esto coincidiría con lo expuesto por la autora paraguaya, quien menciona que el $86,97 \%$ de sus participantes tenían diagnosticada la enfermedad con un tiempo no mayor de 15 años ${ }^{(8)}$. En Chile, Ávila-Jiménez et al. mencionan una prevalencia en el rango de 31 a 50 años. La semejanza puede explicarse por el aumento de los métodos diagnósticos y de las campañas de tamizaje de diabetes que toman importancia como medida de prevención y contingencia contra las enfermedades no transmisibles (entre las cuales está la diabetes) ${ }^{(16)}$.

Ríos González analizó el soporte familiar en pacientes diabéticos, y clasificó esta variable en alto, mediano y bajo. La mayor parte de sus pacientes corresponde a un grupo con apoyo familiar medio, que representa el $93,31 \%$ de los estudiados ${ }^{(8)}$. El encontrar que los pacientes diabéticos tienen un apoyo familiar regular o deficiente se puede justificar por lo descrito por Reynoso-Vázquez et al., quienes enumeran que el trabajo domiciliario del tratamiento de la diabetes tiene una carga familiar en múltiples aspectos ${ }^{(17)}$. En su estudio describen la percepción de apoyo familiar por parte de los pacientes y detallan que la gran mayoría de ellos percibe que nunca son apoyados por los familiares en el aspecto económico, la rutina de ejercicios y cambio de dieta ${ }^{(8)}$; por el contrario, en el estudio que realizamos el $62,00 \%$ de los encuestados tuvo un apoyo familiar adecuado. El reporte de Merodio Pérez indica que la percepción del apoyo familiar de los pacientes diabéticos adultos mayores proviene principalmente del cónyuge, mientras que, en este estudio, el cuidador primario predominante fue el hijo (18). La razón de este contraste sería la diferencia cultural de las dinámicas familiares, dado que en el Perú predominan las familias con cohesión aglutinada y conectada ${ }^{(18)}$.

En un estudio en Pinar del Río, en Cuba, Cuellar Álvarez detalló que las complicaciones más frecuentes en los sujetos estudiados fueron las cardiovasculares $(38,1 \%)$, seguidas de las complicaciones renales $(19,1 \%)$, a diferencia del presente estudio, donde se encontró que el $43,70 \%$ de los participantes no eran portadores de ninguna complicación; y entre los que sí las tenían, el 20,30 \% mostraba más de una, lo que se catalogó como complicaciones múltiples y dejaban en rezago a las complicaciones cardiovasculares y renales. Estas diferencias, descritas en el estudio de Cuellar Álvarez, se explican por el tiempo en el cual se realizó el estudio, que fue entre los años 1992 y 2001 (19). Actualmente, se tiene mayor conocimiento sobre las complicaciones causadas por la diabetes mellitus, su diagnóstico y manejo ${ }^{(20)}$.

Se observa también que la variable rango de edad presenta asociación significativa respecto al control glicémico. Por el contrario, variables como cuidador primario ${ }^{(20)}$, tipo de 
Asociación entre soporte familiar y control glicémico en

pacientes con diabetes mellitus de tipo 2 del Consultorio de

Endocrinología de un hospital nacional

esquema familiar ${ }^{(9)} \mathrm{O}$ incluso nivel de instrucción (nivel de conocimientos) (17), que se asociaron con un buen control glicémico en los estudios citados, no mostraron este resultado en nuestra investigación.

En 2019, Espínola Chamorro ${ }^{(8)}$ encontró que $12,11 \%$ de su muestra tenía mal control glicémico asociado a un bajo apoyo familiar. En nuestro trabajo, el 27,80 \% de la muestra tenía control glicémico asociado a un mal soporte familiar. En ambos estudios se concluye que el paciente con mal control glicémico tienen asociado a él un soporte familiar no adecuado.

Una de las limitaciones de este estudio es el tipo de muestreo utilizado, ya que fue no probabilístico por conveniencia. Se podría esperar que la mayor cantidad de pacientes que asisten al Área de Diabetes del hospital tengan un buen control glicémico y, por el contrario, que aquellos que no asisten tengan un mal control glicémico. Sin embargo, se encontró lo contrario: esto puede deberse a que la mayor parte de pacientes controlados afirmaron que ya no asisten al Área de Diabetes, pero que en su momento lo hicieron. Por otro lado, los pacientes no controlados sí refieren asistir al Área de Diabetes en el presente.

Se concluye que existe asociación entre el soporte familiar que recibe una persona diabética y el control glicémico que maneja. Además, la variable rango de edad demostró una asociación significativa con el control glicémico de los pacientes. Las variables fuertemente asociadas al control glicémico en estudios previos, como cuidador primario o nivel de instrucción, no tuvieron asociación en el presente informe. El hijo en el papel de cuidador primario representa a la mitad de la muestra.

Más del $75 \%$ de los pacientes tiene un tiempo de enfermedad menor a 15 años. Poco menos de la mitad de los participantes tiene complicaciones relacionadas a la diabetes: la mayoría de este grupo presenta más de una, y la más frecuente es el problema neurológico. En el presente estudio, la hipertensión arterial es la comorbilidad más representativa de la diabetes mellitus.

Se recomienda realizar estudios sobre factores de riesgo que pudieran influir en el control glicémico de un paciente diabético, e incentivar y educar a los pacientes diabéticos sobre la importancia de seguir sus controles, de participar en el Programa de Diabetes y asistir de manera regular al Área de Diabetes del hospital. Además de concientizar a los familiares, y sobre todo al cuidador primario, de ser un soporte vital para el paciente diabético y su control.

Fuentes de financiamiento: El artículo ha sido financiado por los autores.

Conflicto de intereses: Los autores declaran no tener ningún conflicto de intereses.
Responsabilidades éticas: El estudio, contó con la autorización de las autoridades pertinentes del Hospital Nacional Dos de Mayo y con la aprobación del Comité de Ética de la FMH-USMP y del HNDM. Forma parte de la tesis que será presentada por Carlos Abuhadba Miranda. La Dra. Nora Espíritu fue asesora metodológica y el Dr. Dante Gamarra, asesor temático.

Confidencialidad de los datos: Se respetaron las normas de la buena práctica clínica y se cumplieron las normas éticas estipuladas en la declaración de Helsinki. El instrumento de recolección de datos junto con la escala FACES III fueron utilizados exclusivamente para la investigación y descartados después del estudio. Derecho a la privacidad y consentimiento informado: Los instrumentos de recolección de datos fueron codificados y se mantuvo la estricta confidencialidad de los participantes, la participación fue voluntaria y no hubo costos adicionales por participar en el estudio.

Aportación original e importancia del artículo: La diabetes mellitus tipo 2 es una enfermedad que ha ido aumentando su prevalencia e incidencia a lo largo de todo el mundo en los últimos años. Es una enfermedad que es asiento de múltiples comorbilidades como la enfermedad cerebrovascular, pie diabético, poli neuropatías, infecciones, etc., que ocasionan altas tasas de mortalidad y discapacidad, las mismas que tienen altos costos en su tratamiento y rehabilitación.

Es aquí donde la prevención primaria adquiere relevancia, un buen soporte familiar, acompañado de adecuada educación al paciente, puede mejorar la evolución drásticamente y retrasar el avence de la enfermedad.

\section{REFERENCIAS BIBLIOGRÁFICAS}

1. Mathers CD, Loncar D. Projections of global mortality and burden of disease from 2002 to 2030. PLoS Med. 2006; 3(11): e442.

2. Ministerio de Salud. Guía de Práctica Clínica para el Diagnóstico, Tratamiento y Control de la Diabetes Mellitus Tipo 2 en el Primer Nivel de Atención [Internet]. Dirección de Prevención de Enfermedades No Transmisibles y Oncológicas: MINSA; Lima. 2016. Disponible en: http://bvs.minsa.gob.pe/local/MINSA/3466.pdf

3. Seclen SN, Rosas ME, Arias AJ, Huayta E, Medina CA. Prevalence of diabetes and impaired fasting glucose in Peru: report from PERUDIAB, a national urban population-based longitudinal study. BMJ Open Diabetes Res Care. 2015; 3(1): e000110.

4. Instituto Nacional de Estadística e Informática. Enfermedades No Transmisibles y Transmisibles, 2017 [Internet]. INEl: Lima, Perú. 2018. Disponible en: https://www.inei.gob.pe/media/MenuRecursivo/ publicaciones_digitales/Est/Lib1526/index.html

5. Ministerio de Salud. Carga de enfermedad en el Perú: Estimación de los años de vida saludables perdidos 2012 [Internet]. Dirección General de Epidemiología: Ministerio de Salud; Lima. 2014. Disponible en: http://bvs.minsa.gob.pe/local/MINSA/2993.pdf

6. Gonzales-Grández NN, Rodríguez-Lay EG, Manrique-Hurtado 
H. Características clínicas y factores asociados a morbilidad intrahospitalaria en los pacientes con diabetes mellitus tipo 2. Rev Soc Peru Med Interna. 2013; 26(4): 159-65.

7. Rodríguez MCC, Ramos RMMO, Díaz OH. La familia en el cuidado de la salud. Rev Med Electrón. 2014; 36(4): 462-72.

8. Ríos González CM, Espinola Chamorro CE. Apoyo familiar y control glicémico en pacientes diabéticos de un Hospital de III Nivel de atención de Paraguay. Rev Nac (Itauguá). 2020; 12(1): 28-41.

9. Fundación redGDPS. Guía de diabetes tipo 2 para clínicos: Recomendaciones de la RedGDPS [Internet]. España; 2018. Disponible en: http://www.redgdps.org/gestor/upload/colecciones/Guia\%20 DM2_baja_\%20modificaciones.pdf

10. Zegarra GSC. Relación entre el nivel de apoyo familiar en el cuidado y la calidad de vida de los pacientes con diabetes mellitus tipo 2, atendidos en la consulta externa del Hospital Nacional Guillermo Almenara Irigoyen, Lima, Perú. Rev Científica Cienc Salud. 2013; 6(2): 7-14

11. Vivero MGC. Funcionamiento familiar, control glucémico y la relación con el esultado de la prueba de monofilamento como indicador de neuropatía periférica en los pacientes con diabetes tipo 2 de la cClínica de Crónicos Metabólicos del Centro de Salud los Rosales, octubre a noviembre del Año 2016 [Tesis]. Quito: Pontificia Universidad Católica del Ecuador. Facultad de Medicina; 2016.

12. García-Morales G, Rodríguez-Pascual A, Garibo-Polanco RE. Apoyo familiar y control glucémico en pacientes con diabetes tipo 2 en una unidad de medicina familiar de Acapulco, Guerrero, México. Aten Fam. 2018; 25(1). 27-31.

13. Sánchez Basilio NA. Relación entre funcionalidad familiar y control glucémico en pacientes con diabetes mellitus tipo 2 [Tesis]. Veracruz: Universidad Veracruzana. Facultad de Medicina; 2013.

14. Cárdenas RVT. Cohesión y adaptabilidad familiar y su relación con el rendimiento académico [Tesis]. Lima: Universidad Nacional Mayor de San Marcos. Facultad de Psicología; 2004.

15. Lope PLC. Asociación entre funcionalidad y red de apoyo familiar en el control glicémico de pacientes diabéticos tipo 2, atendidos en el programa de enfermedades no trasmisibles. Hospital III EsSaludGrau - 2014 [Tesis]. Lima: Universidad Nacional Mayor de San Marcos. Facultad de Medicina Humana; 2015.

16. Ávila-Jiménez L, Cerón OD, Ramos-Hernández RI, Velázquez LL. Asociación del control glicémico con el apoyo familiar y el nivel de conocimientos en pacientes con diabetes tipo 2. Rev Méd Chile. 2013; $141(2):$ 173-80.

17. Reynoso-Vázquez J, Hernández-Rivero E, Martínez-Villamil $M$, Zamudio-López JL, Islas-Vega I, Pelcastre-Neri A, et al. La atención en casa: El apoyo familiar en el control glicémico en pacientes con diabetes mellitus tipo 2. Hosp Domic. 2020; 4(4): 199-207.

18. Merodio Pérez M, Acuña VR, Serrano AM. Percepción del apoyo familiar y dificultades relacionadas con la diabetes en el adulto mayor. Horiz Sanit. 2015; 14(1): 14-20.

19. Álvarez RC, Delgado MT, Chang AMJ, Oca SLM. Comportamiento de la mortalidad por diabetes mellitus. Provincia Pinar del Río. 1992-2001. Rev Cubana Endocrinol. 2002; 13(Supl): 79

20. Zegarra DV, Jesús AP. Cohesión, adaptabilidad y composición familiar en adolescentes del Callao, Perú. Propósitos y Representaciones. 2017; 5(2): 21-64.
Correspondencia:

Carlos Antonio Abuhadba Miranda.

Dirección: Calle Cantuarias 355 Dpto. 203. Miraflores, Lima. Teléfono: +51984008 626 .

Correo electrónico: carlos.abuhadba7298@gmail.com

Recibido: 13 de abril de 2021.

Evaluado: 05 de julio de 2021.

Aprobado: 18 de septiembre de 2021.

(c) La revista. Publicado por Universidad de San Martín de Porres, Perú. (c) ${ }_{\text {BY }}$ Licencia de Creative Commons Artículo en acceso abierto bajo términos de Licencia Creative Commons Atribución 4.0 Internacional. (http://creativecommons.org/licenses/by/4.0/)

ORCID iDs

Carlos Abuhadba Miranda

Nora Espíritu

Dante Gamarra Gonzáles

(- https: / / orcid.org/0000-0003-1716-0919

(1) https: / / orcid.org/0000-0002-6340-0285

(1) https: / / orcid.org/0000-0002-1166-7121 